\title{
HARDY INEQUALITIES
}

\author{
ANDREAS WANNEBO
}

(Communicated by John B. Conway)

Abstract. Here the following Hardy inequalities are studied

$$
\sum_{k=0}^{m-1} \int \frac{\left|\nabla^{k} u\right|^{p} d_{j \Omega}(x)^{t}}{d_{j \Omega}(x)^{(m-k) p}} d x \leq A_{\Omega} \int\left|\nabla^{m} u\right|^{p} d_{j \Omega}(x)^{t} d x
$$

for $u \in C_{0}^{\infty}(\Omega)$, an open proper subset of $\mathbf{R}^{N}$ and $t<t_{0}$, some small positive $t_{0}$. This inequality has previously been shown to hold for bounded Lipschitz domains. The question discussed is, How general can $\Omega$ be and allow the same inequality? A sufficient condition is given in the form of a local Maz' ${ }^{\prime}$ a capacity condition. In $\mathbf{R}^{2}$, or generally if $p>N-1$, this is satisfied for any $\Omega$ which is deformable to a point. Furthermore, if $p>N$ the condition is satisfied for all $\Omega$.

\section{INTRODUCTION}

The original Hardy inequality can easily be written in the following form:

$$
\int_{0}^{\infty}|u(s)|^{p} s^{-p+a} d s \leq\left(\frac{p}{p-1-a}\right)^{p} \int_{0}^{\infty}\left|u^{\prime}(s)\right|^{p} s^{a} d s .
$$

Actually there are two instances of the inequality. The one of interest here has the conditions that $1<p<\infty, a<p-1, u$ is absolutely continuous and furthermore $u(0)=0$. This inequality was given by G. H. Hardy and others in [3].

This inequality has been generalized in many ways. The generalization considered here is to functions in $C_{0}^{\infty}(\Omega)$ with $\Omega$ an open and proper subset of $\mathbf{R}^{N}$. The studied inequality then reads

$$
\sum_{k=0}^{m-1} \int \frac{\left|\nabla^{k} u\right|^{p} d_{\partial \Omega}(x)^{t}}{d_{\partial \Omega}(x)^{(m-k) p}} d x \leq A_{\Omega} \int\left|\nabla^{m} u\right|^{p} d_{\partial \Omega}(x)^{t} d x,
$$

where $\nabla^{k} u$ is the vector of all derivatives of order $k$ and $\left|\nabla^{k} u\right|$ is the Euclidean length of that vector. Furthermore $d_{\partial \Omega}(x)$ is the distance from $x$ to $\partial \Omega$.

Received by the editors June 19, 1988.

1980 Mathematics Subject Classification (1985 Revision). Primary 26D15, 46E35, 35J30. 
The inequality is true for nice regions RHS and LHS exponents are then optimal for $t<p-1$. The question raised here is, How general can a domain $\Omega$ be and still allow this optimal inequality? So far there is a simple sufficient condition only if $t<t_{0}$ for some $t_{0}>0$, and this is the case that will be treated here.

Every open proper subset $\Omega$ of $\mathbf{R}^{N}$ has a decomposition in closed cubes with diameters $2^{n}$ for $n \in \mathbf{Z}$ such that their interiors are disjoint and $\operatorname{diam} Q \leq$ $\operatorname{dist}\left(Q, \Omega^{c}\right) \leq 4 \operatorname{diam} Q$ for the cubes $Q$ in the decomposition. Such a decomposition is called a Whitney decomposition of the region $\Omega$. See E. M. Stein [11, p. 167] for reference.

Now we describe the condition. A local capacity of condenser type, defined by V. G. Maz'ja, of part of the complement of the region as seen from a Whitney cube is bounded away from zero by a positive constant. It is not known whether this condition is also necessary.

In $\mathbf{R}^{2}$ this condition and inequality (1) are true for any region $\Omega$ which can be deformed to a point, and generally it is true if $\Omega$ is deformable to a point and $p>N-1$. Furthermore, inequality (1) is true if $p>N$. The reasons for this behavior are that the Bessel capacity $C_{1, p}$ of a continuum of unit diameter is greater than a positive constant if $p>N-1$, and the same capacity of a point is a positive constant if $p>N$.

There have been several results given for inequality (1). The earliest(?) was by $J$. Nečas [10, Theorem 1.6]. This result is that the inequality $(1)$ is true for bounded Lipschitz domains and with parameters $1<p$ and $t<p-1$.

In [1] A. Ancona gave a simple proof of the inequality (1) for $p>N, t=0$, and any open bounded $\Omega$ in $\mathbf{R}^{N}$. With this value of $p$, the Bessel capacity $C_{1, p}$ of a point is positive, as above, and this is why geometrical considerations are not difficult in his case.

Define $W^{m, p}(\Omega)$ as the Banach space, which is the completion of $C^{\infty}(\Omega)$, $\Omega$ open in $\mathbf{R}^{N}$, in the norm

$$
\|u\|_{\mathcal{W}^{\prime m, p}(\Omega)}=\sum_{k=0}^{m}\left(\int_{\Omega}\left|\nabla^{k} u\right|^{p} d x\right)^{\frac{1}{p}} .
$$

Also define $W_{0}^{m, p}(\Omega)$ as the completion of $C_{0}^{\infty}(\Omega)$ in the same norm. Define $W^{m, p, t}(\Omega)$ as the completion of $C^{\infty}(\Omega)$ in the norm

$$
\|u\|_{\boldsymbol{H}^{\prime m, \cdots, \lambda^{\prime}(\Omega)}}=\sum_{k=0}^{m}\left(\int\left|\nabla^{k} u\right|^{p} d_{i, \Omega}(x)^{t} d x\right)^{\frac{1}{p}} .
$$

Also define $W_{0}^{m, p, t}(\Omega)$ as the completion of $C_{0}^{\infty}(\Omega)$ in the same norm.

A. Ancona [1] studied functions in the Sobolev space $W_{0}^{m \cdot p}(\Omega)$ which are the difference of two positive functions in the same space. This question is related to inequality (1), with $t=0$. A further treatment is given in [13]. The question was raised by $\mathrm{H}$. Brezis and $\mathrm{F}$. Browder, see [2]. 
The importance of capacities of Hardy inequalities for $C_{0}^{\infty}(\Omega)$ has previously been shown by V. G. Maz'ja, see [7, chap. 2]. The Maz'ja result is very general; if it is restricted to $L^{p}$-norms we get the following result.

Define for a compactum $F$

$$
(p, \Phi)-\operatorname{cap}(F, \Omega)=\inf \left\{\int_{\Omega}[\Phi(x, \nabla u)]^{p} d x: u \in M(F, \Omega)\right\},
$$

where $\Phi(x, \zeta)$ is a continuous function of $x \in \Omega$ and $\zeta \in \mathbf{R}^{N}$, which is nonnegative and positive homogeneous of the first degree with respect to $\zeta$. Furthermore,

$$
M(F, \Omega)=\left\{u \in C_{0}^{\infty}(\Omega): u \geq 1 \text { on } F\right\} .
$$

The Maz'ja result now reads (except for the estimate of the constant) that the following two statements are equivalent.

There is a constant $\beta$ such that for every compactum $F$ in $\Omega$

$$
\mu(F) \leq(p, \Phi)-\operatorname{cap}(F, \Omega) \cdot \beta .
$$

There exists a constant $C$ such that for every $u \in C_{0}^{\infty} .(\Omega)$ it is true that

$$
\int_{\Omega}|u|^{p} d \mu \leq C \int_{\Omega}[\Phi(x, \nabla u)]^{p} d x .
$$

It seems to be difficult to use this result in the cases considered here, since all compacta $F \subset \Omega$ are involved and the capacity-measure comparison seems difficult to verify.

E. W. Stredulinsky [12] also presents a wealth of material on inequalities in weighted spaces.

The case with $t<p$ in inequality (1) is treated in [13], where the sufficient condition is more complicated. For instance, a concept called the local dimension of the boundary puts a restriction on the domains.

The proof of the main result is based on a theorem by V. G. Maz'ja which is a Poincare inequality for functions defined in a cube. This theorem follows from a weak Poincare inequality for a cube. Very few restrictions on the choice of norm are needed for this to be true. Thus the result can be generalized to norms other than $L^{p}$. Furthermore, the procedures used in the proof are not specific for $L^{p}$ and there are thus generalizations of the main result.

The applications of inequality (1) are many, for instance in the theory of PDE's. For some applications see [5] and [13]. A. Kufner gives a theorem as application [5, p. 101]. There the requirement was that $\Omega$ should be bounded Lipschitz. He used the Nečas result, above; and if we instead use inequality (1), then we get a similar theorem. But the interval $I$ appearing here is not the same as his, and there is only a weak condition on $\Omega$. We give this only as an example.

Let $\mathscr{L}$ be a differential operator of order $2 k$ (with $k$ a positive integer) given by the expression

$$
\mathscr{L} u(x)=\sum_{|\cdots| .|\beta| \leq k}(-1)^{|n|} D^{\prime \prime}\left(a_{\cdots, \beta}(x) D^{\beta} u(x)\right) .
$$


Furthermore, let the bilinear form associated to be the differential operator $\mathscr{L}$ and open domain in $\mathbf{R}^{N}, \Omega$, be

$$
a_{\mathscr{L}, \Omega}(u, v)=\sum_{|\alpha|,|\beta| \leq k} \int_{\Omega} a_{\alpha, \beta}(x) D^{\alpha} v(x) D^{\beta} u(x) .
$$

The operator $\mathscr{L}$ is said to be elliptic if

$$
a_{\mathscr{L}, \Omega}(u, u) \geq c\|u\|_{k, 2}^{2}
$$

for all $u \in W_{0}^{m, p}(\Omega)$.

Let $\Omega$ be in $\mathbf{R}^{N}$ and open. Suppose that $u_{0} \in W^{k, 2, t}(\Omega)$ and $F \in$ $\left(W_{0}^{k, 2,-t}\right)^{*}$. The asterisk denotes taking the dual space. Then $u$ is said to be a weak solution of the Dirichlet problem for $\mathscr{L}$ if

(i) $u-u_{0} \in W_{0}^{k, 2, t}(\Omega)$ and if

(ii) for all $v \in C_{0}^{\infty}(\Omega)$ we have

$$
a_{\mathscr{L}, \Omega}(u, v)=\langle F, v\rangle .
$$

We have the result that if $\Omega$ is a bounded, open, and proper subset of $\mathbf{R}^{2}$, which is deformable to a point, if $\mathscr{L}$ is an elliptic differential operator defined as above, and if $a_{\alpha, \beta}(x)$ are bounded functions, then there is an open interval $I$ which contains zero such that for $t \in I$ there exists one unique weak solution to the Dirichlet problem for $\mathscr{L}$ in $\Omega$ in the sense defined above. Furthermore,

$$
\|u\|_{W^{k, 2.1}(\Omega)} \leq c\left(\left\|u_{0}\right\|_{W^{k, 2.1}(\Omega)}+\|F\|_{\left(W^{k, 2,-1}\right)^{*}}\right)
$$

for $c$ a positive constant.

\section{NOTATION AND PRELIMINARIES}

For $\Omega$ open in $\mathbf{R}^{N}$ and with a Whitney decomposition $\mathscr{F}$ we choose a closed cube $R_{Q}$ belonging to $Q \in \mathscr{F}$ in a nonunique way as follows. Pick one of the points in $\partial \Omega$ at minimum distance from $Q$, then take this point as center for the cube $R_{Q}$, which has the same orientation as $Q$, but has minimal diameter under the condition that $Q \subset R_{Q}$.

Given an $R_{Q}$, we denote the cube with the same center and dilated to unit diameter with $R_{\widetilde{Q}}$; and if $u$ is a function defined in $R_{Q}$, we denote the function arising from the same dilation with $\tilde{u}$. Furthermore $\widetilde{\Omega}$ is defined in the same way if it is clear from the context which $R_{Q}$ is considered.

Let $A$ denote a generic positive constant which may change even in the same string of inequalities.

The starting point of the proof of my result is a theorem by V. G. Maz'ja. His theorem is a kind of Poincare inequality, given in [6, §5] and [7, p. 414].

Definition (Maz'ja). Let $1 \leq p<\infty$ and $m$ be a positive integer. Let $K$ be a closed subset of the closed cube $Q_{0}$ in $\mathbf{R}^{N}$ with unit diameter. Let $\mathscr{P}_{m-1}$ be the set of polynomials of degree less than or equal to $m-1$ with $L^{p}\left(Q_{0}\right)$ 
norm equal to one. Let $T_{P}$ be the subset of $C_{0}^{\infty}\left(2 Q_{0}\right)$ consisting of functions $v$ such that $v=P$ in a neighborhood of $K$. Then the Maz'ja capacity of $K$ is defined as

$$
\gamma_{m, m-1, p}\left(K, 2 Q_{0}\right)=\inf _{P \in \mathscr{S}_{m-1}} \inf _{m \in T_{P}} \int\left|\nabla^{m} v\right|^{p} d x .
$$

Theorem A (Maz' $\left.{ }^{\prime} \mathrm{ja}\right)$. Let $1 \leq p<\infty$ and let $m$ be a positive integer. If $K$ is a closed subset of $Q_{0}$, a closed cube in $\mathbf{R}^{N}$ with unit diameter, then the condition $\gamma_{m, m-1, p}\left(K, 2 Q_{0}\right)>0$ implies the following inequality for $u \in C_{0}^{\infty}\left(K^{c}\right)$

$$
\sum_{k=0}^{m-1} \int_{Q_{0}}\left|\nabla^{k} u\right|^{p} d x \leq C_{K} \int_{Q_{0}}\left|\nabla^{m} u\right|^{p} d x
$$

with $C_{K}=c \cdot\left(\gamma_{m, m-1, p}\left(K, 2 Q_{0}\right)\right)^{-1}$, where $c$ depends only on $N, m$, and $p$.

Remark. The theorem given by Maz' ja has only the zero-order term in the LHS, but it is possible to go through his calculations, using the lemma in [7, p. 22] and get this result.

Let $\Omega_{n}$ be the (interior of the) subset of $\Omega$ built by the Whitney cubes with diameter less than or equal to $2^{-n}$.

Lemma 1. Let $f$ and $g$ be functions defined in $\mathbf{R}^{N}$ such that $f, g=0$ in $\Omega^{c}$. Suppose that $\Omega$ has a Whitney decomposition $\mathscr{F}$. Assume furthermore that

$$
\int_{Q} f d x \leq \int_{R_{Q}} g d x
$$

for all cubes $Q \in \mathscr{F}$.

Then

$$
\int_{\Omega_{n}} f d x \leq A \int_{\Omega_{n-a}} g \cdot(\rho(x)-n+a+1) d x,
$$

where $\rho(x)$ is defined a.e. as $-{ }^{2} \log \operatorname{diam} Q$ for $Q \in \mathscr{F}$ if $x \in Q$ and $a$ is a constant depending only on $N$. The value of a can be taken as $a=$ $\left.\Gamma^{2} \log (4 \sqrt{N}+1+\varepsilon)\right\rceil$. Furthermore $A$ depends only on $N$.

Proof. The value of $a$ has been chosen in order to ensure that if $\operatorname{diam} Q^{\prime}>$ $2^{-k+a}$ and $\operatorname{diam} Q \leq 2^{-k}$, then $Q^{\prime} \cap R_{Q}$ is empty. This is the consequence of the following argument. The quotient $\operatorname{diam} R_{Q}$ over $\operatorname{diam} Q$ takes its largest value if $Q$ is situated exactly at the middle of a face of $R_{Q}$ and $4 \operatorname{diam} Q=$ $\operatorname{dist}\left(Q, \Omega^{c}\right)$, since if we move $Q$ about in $R_{Q}$ following a face we get an equal or longer distance otherwise. Then $Q$ has to be made larger in order to satisfy the Whitney cube condition $4 \operatorname{diam} Q \geq \operatorname{dist}\left(Q, \Omega^{c}\right)$. But here we have assumed in the definition of $R_{Q}$ that $\operatorname{dist}\left(Q, \Omega^{c}\right)=\operatorname{dist}\left(Q, \operatorname{center}\left(R_{Q}\right)\right)$.

To calculate $a$ we note that in the extremal case above, the diameter of $R_{Q}$ becomes $2(4 \operatorname{diam} Q+\operatorname{diam} Q / \sqrt{N}) \sqrt{N}$, i.e. the quotient is $2(4 \sqrt{N}+1)$. It follows that $R_{Q}$ touches no cube with diameter larger than $(4 \sqrt{N}+1)$ diam $Q$, 
since we have the Whitney cube condition $\operatorname{diam} Q \leq \operatorname{dist}\left(Q, \Omega^{c}\right)$. Hence take $\left.a=\Gamma^{2} \log (4 \sqrt{N}+1+\varepsilon)\right\rceil$, any $\varepsilon>0$.

Now we sum the inequalities coming from cubes $Q$ in $\mathscr{F}$ with diameter less than or equal to $2^{-n}$. The consequence is that a point in $\Omega_{n}$ belonging to a Whitney cube with diameter $2^{-k}$ will be counted exactly once a.e. in the LHS we then will get, but in the RHS there will be overlap from many $R_{Q}$ 's, that will give an increasing weight factor in the integral when $k$ goes to $\infty$, i.e. $d_{\partial \Omega}(x)$ goes to 0 . We shall now determine that weight factor.

Summing the given relations between the functions $f$ and $g$ for $Q$ 's such that $\operatorname{diam} Q \leq 2^{-n}$ gives

$$
\int_{\Omega_{n}} f d x=\sum_{\operatorname{diam} Q \leq 2^{-n}} \int_{\Omega_{n}} f \cdot \chi_{Q} d x \leq \int_{\Omega_{n-a}} \sum_{\operatorname{diam} Q \leq 2^{-n}} g \cdot \chi_{R_{Q}} d x .
$$

Now trivially

$$
\sum_{\operatorname{diam} Q \leq 2^{-n}} g(x) \chi_{R_{Q}}(x)=g(x) \cdot \sum_{\operatorname{diam} Q \leq 2^{-n}} \chi_{R_{Q}}(x),
$$

and it is only needed to evaluate the function

$$
s(x)=\sum_{\operatorname{diam} Q \leq 2^{-n}} \chi_{R_{Q}}(x) .
$$

Suppose $x$ is the interior of $Q^{\prime}$, where $\operatorname{diam} Q^{\prime}=2^{-k}$. Then we estimate $s(x)$ from the above. It suffices to estimate the number of $Q^{\prime}$ s such that $R_{Q} \cap Q^{\prime}$ is nonempty. If $Q^{\prime}$ meets $R_{Q}$, then $\operatorname{dist}\left(Q^{\prime}, \Omega^{c}\right) \leq \operatorname{diam} R_{Q}$. Hence by the Whitney cube condition $\operatorname{diam} Q^{\prime} \leq \operatorname{diam} R_{Q}$.

Now cube $Q$ lies in a ball with radius $\operatorname{diam} R_{Q}+\frac{1}{2} \operatorname{diam} Q^{\prime}$. But this is less than or equal to $(2(4 \sqrt{N}+1)+4 \sqrt{N}+1) \cdot \operatorname{diam} Q$.

By a volume consideration, only a fixed number of $Q$ 's with the same diameter can be in this ball. Call this number $A(N)$ since it depends only on $N$ according to this calculation. It follows that if $x \in Q^{\prime}$ then $s(x)$ is at most

$$
A(N)(a+k-n+1)=A(N)\left(-{ }^{2} \log \left(\operatorname{diam} Q^{\prime}\right)-n+a+1\right),
$$

since each value of $r$ between $n$ and $k-a$ contributes with at most counting $A(N)$ times. The lemma follows.

Lemma 2. Let $\alpha$ be greater than zero and small. Let $s$ be greater than $s_{a r}$ for some large $s_{1}$. Then

$$
\sum_{n=a}^{s} e^{n+r} \sim \int_{a}^{s} e^{n a r} d n \sim \frac{e^{s i r}}{\alpha}
$$

where $\alpha$ is a small constant.

The easy proof of this simple statement is omitted. 
Lemma 3. Let $\alpha$ be greater than zero, a be a fixed positive constant, $s$ be greater than $s_{\alpha}$ for some $s_{\alpha}$ large, and $\alpha$ be small. Then it is true that

$$
\sum_{0}^{s} e^{n \alpha x}(s-n+a+1) \sim \int_{0}^{s} e^{n \alpha}(s-n+a+1) d n \sim \frac{e^{s \alpha}}{\alpha^{2}} .
$$

The argument for this is of the same type as for the lemma above, though the situation is more complicated. To understand the first step, observe that the standard estimate of sums with integrals used the monotonicity of the function involved. This function has only one maximum; hence standard estimates can be done piecewise. What happens is that the sum "equals" the integral plus or minus the values at three points on the curve, endpoints, and maximum. But when $s_{c}$ is large these point values are neglible compared with the value of the whole integral.

The integral gives, after partial integration, the RHS as the dominating term if $\alpha$ is small.

\section{THE MAIN RESULT}

Theorem. Let $\Omega$ be an open set in $\mathbf{R}^{N}$. Let $\mathscr{F}$ be some Whitney decomposition of $\Omega$. Let $1<p<\infty$, and let there be a constant $b>0$ such that for every $Q$ in $\mathscr{F}$ it is true that

$$
\gamma_{m, m-1, p}\left(\left(\widetilde{\Omega}^{c} \cap R_{\widetilde{Q}}\right), 2 R_{\widetilde{Q}}\right) \geq b .
$$

Then

$$
\sum_{k=0}^{m-1} \int \frac{\left|\nabla^{k} u\right|^{p} d_{j \Omega}(x)^{t}}{d_{j \Omega}(x)^{(m-k) p}} d x \leq \frac{A}{b} \int\left|\nabla^{m} u\right|^{p} d_{\partial \Omega}(x)^{t} d x
$$

for every $u \in C_{0}^{\infty}(\Omega)$ and for $t<t_{0}$, some $t_{0}>0$, where $A$ depends on $m, p$, $N$, and $t$.

Remark. Estimates of both $A$ and $t_{0}$ can be made from the calculations in the proof of the main theorem and of the Maz'ja theorem if a value of $C$ in the inequality

$$
\sum_{k=0}^{m-1} \int_{Q}\left|\nabla^{k} u\right|^{p} d x \leq C \int_{Q}\left|\nabla^{m} u\right|^{p} d x
$$

is given.

Remark. It should be noted that the result built on $m=1$, and then on induction on $m$, cannot cover all the $\Omega$ 's covered by this theorem, since the Maz'ja capacities are polynomial in nature.

Proof. The condition in the theorem and the result of Theorem A implies that

$$
\sum_{k=0}^{m-1} \int_{\widetilde{Q}} \frac{\left|\nabla^{k} \tilde{u}\right|^{p}}{d_{i \widetilde{\Omega}}(x)^{(m-k) p}} d x \leq 2^{m p} \sum_{k=0}^{m-1} \int_{R_{\widetilde{Q}}}\left|\nabla^{k} \tilde{u}\right|^{p} d x \leq \frac{A}{b} \int_{R_{\widetilde{Q}}}\left|\nabla^{m} \tilde{u}\right|^{p} d x .
$$


Here we have used that $d_{\partial \widetilde{\Omega}}(x) \geq \frac{1}{2}$, since by the Whitney cube property we have that $\operatorname{diam} Q / \operatorname{diam} R_{Q} \leq \frac{1}{2}$.

Now we dilate back this result, mapping $R_{\widetilde{Q}}$ back on $R_{Q}$ etc. Observe that a dilation is a change of the independent variable by a positive factor, say $\lambda$, and that this transforms $\nabla^{k} \tilde{u}$ into $\lambda^{k} \cdot \nabla^{k} u, d_{\partial \widetilde{\Omega}}$ into $\lambda^{-1} \cdot d_{\partial \Omega}(x)$ (if $\lambda=\operatorname{diam} R_{Q} / \operatorname{diam} R_{\widetilde{Q}}$ i.e.), and $d x$ into $\lambda^{-N} \cdot d x$.

$$
\sum_{k=0}^{m-1} \int_{Q} \frac{\left|\nabla^{k} u\right|^{p}}{d_{\partial \Omega}(x)^{(m-k) p}} d x \leq \frac{A}{b} \int_{R_{Q}}\left|\nabla^{m} u\right|^{p} d x .
$$

This is true since both sides are dilation homogenous and to the same degree.

Now this is the requirement for Lemma 1. We get the following result:

$$
\sum_{k=0}^{m-1} \int_{\Omega_{n}} \frac{\left|\nabla^{k} u\right|^{p}}{d_{\partial \Omega}(x)^{(m-k) p}} d x \leq \frac{A}{b} \int_{\Omega_{n-a}}\left|\nabla^{m} u\right|^{p} \rho(x)-n+a+1 d x .
$$

This formulation is not what we wanted, because of the annoying $\rho(x)$-factor in the RHS. The factor is logarithmic in $d_{\partial \Omega}(x)$. What is needed instead is a bootstrap procedure to get rid of the $\rho(x)$-factor and still keep an inequality.

Take the last inequality and multiply it with a factor $e^{n \times x}$, where $\alpha$ is to satisfy a condition to be determined later, and sum from $n=a$ to $n=\infty$. If we take the sums over $n$ together with the $e^{n / x}$ factors under the integral sign, we can estimate these new integrands.

For $x \in \Omega_{s} \backslash \Omega_{s+1}$ the LHS integrands look like

$$
\sim \sum_{n=a}^{s} e^{n \kappa} \frac{\left|\nabla^{k} u\right|^{p}}{d_{\partial \Omega}(x)^{(m-k) p}}
$$

and the RHS integrand looks like

$$
\sim \sum_{n=0}^{s} e^{n \times}(s-n+a+1)\left|\nabla^{m} u\right|^{p} .
$$

By Lemma 2 we have that $\sum_{n=a}^{s} e^{n \kappa} \sim \frac{1}{\alpha} e^{s \kappa}$ if $s \geq s_{\alpha}$, where $s_{\alpha}$ is large enough. By Lemma 3 we have that

$$
\sum_{n=0}^{s} e^{n x}(s-n+a+1) \sim \frac{e^{s x}}{\alpha^{2}}
$$

if $\alpha$ is small and $s \geq s_{\alpha}$, where $s_{\alpha}$ is large enough.

What has happened to the LHS and the RHS after these estimates have been made is that an extra weight has appeared which is the same on both sides. Furthermore, an extra $\frac{1}{\alpha}$ has appeared in the RHS constant. We now further explain this phenomenon.

To simplify the discussion we assume that $u$ has support in $\Omega_{s k}$ and observe that

$$
e^{s \kappa}=2^{s^{2} \log e^{*} \gamma} \sim d_{j \Omega}(x)^{-\beta},
$$


where $\beta={ }^{2} \log e \cdot \alpha$, since $2^{-s} \sim d_{\partial \Omega}(x)$.

$$
\sum_{k=0}^{m-1} \int \frac{\left|\nabla^{k} u\right|^{p} d_{j \Omega}(x)^{-\beta}}{d_{\partial \Omega}(x)^{(m-k) p}} d x \leq \frac{A_{0}^{p}}{\alpha b} \int\left|\nabla^{m} u\right|^{p} d_{\partial \Omega}(x)^{-\beta} d x .
$$

Contemplating the result so far, it is clear that the obtained inequality is invariant under dilation. This has the effect that the assumption just made on $u$ is no limitation, since if the assumption is not satisfied we dilate $u$ to make it true. Then we get the inequality and it only remains to dilate back.

So far the argument covers all small values of $\beta$. In order to get arbitrary large values of $\beta$, we calculate the same weighted summation, with the last inequality as starting point. It is seen then that if the process is iterated some number of times, the case with any given $t<0$ in the theorem is covered in a finite number of steps.

To do the final step and get the inequality for $0 \leq t<t_{0}, t_{0}>0$, we observe that $d_{\partial \Omega}(x) \sim \tilde{d}_{\partial \Omega}(x)$, where $\tilde{d}_{\partial \Omega}(x)$ is the regularized distance function, see $[11$, p. 171]. This regularized distance function is infinitely many times differentiable in $\Omega$ and has the property that $\left|D^{\gamma} \tilde{d}_{i \Omega}(x)^{\delta}\right| \leq A \delta \tilde{d}_{i \Omega}(x)^{\delta-|y|}$. Here $\delta$ is assumed negative and $\gamma \leq$ const . (The chain rule has also been used.) Here $A$ is independent of $\Omega$ and $\delta$.

Now we make a change of the dependent variable $u$ by putting $v=u$. $\tilde{d}_{i \Omega \Omega}(x)^{-(\beta+t) / p}$. Using repeated differentiations and the triangle inequality, we get the following inequality.

(3)

$$
\begin{aligned}
& \left(\int \frac{\left|\nabla^{k} v\right|^{p} \tilde{d}_{i \Omega}(x)^{t}}{\tilde{d}_{i \Omega \Omega}(x)^{(m-k) p}} d x\right)^{\frac{1}{p}} \\
& \leq A\left(\int \frac{\left|\nabla^{k} u\right|^{p} \tilde{d}_{\partial \Omega}(x)^{-\beta}}{\tilde{d}_{i \Omega}(x)^{(m-k) p}} d x\right)^{\frac{1}{p}}+A \frac{\beta+t}{p} \sum_{r=0}^{k}\left(\int \frac{\left|\nabla^{r} u\right|^{p} \tilde{d}_{j \Omega}(x)^{-\beta}}{\tilde{d}_{i \Omega \Omega}(x)^{(m-r) p}} d x\right)^{\frac{1}{p}} .
\end{aligned}
$$

We sum over $k$ and get

$$
\sum_{k=0}^{m-1}\left(\int \frac{\left|\nabla^{k} v\right|^{p} \tilde{d}_{j \Omega}(x)^{\prime}}{d_{i \Omega}(x)^{(m-k) p}} d x\right)^{\frac{1}{p}} \leq A \sum_{k=0}^{m-1}\left(\int \frac{\left|\nabla^{k} u\right|^{p} \tilde{d}_{i \Omega}(x)^{-\beta}}{\tilde{d}_{i \Omega}(x)^{(m-k) p}} d x\right)^{\frac{1}{\prime}} .
$$

As in the calculation in inequality (3), from the triangle inequality we obtain

$$
\begin{aligned}
A\left(\int\left|\nabla^{m} v\right|^{p} \tilde{d}_{i \Omega}(x) d x\right)^{\frac{1}{p}} \geq & \left(\int\left|\nabla^{m} u\right|^{p} \tilde{d}_{i \Omega \Omega}(x)^{-\beta} d x\right)^{\frac{1}{p}} \\
& -A_{1} \frac{\beta+t}{p} \cdot \sum_{k=0}^{m-1}\left(\int \frac{\left|\nabla^{k} u\right|^{p} \tilde{d}_{i \Omega \Omega}(x)^{-\beta}}{\tilde{d}_{i \alpha \Omega}(x)^{(m-k) p}} d x\right)^{\frac{1}{p}} .
\end{aligned}
$$


Now we observe that in the RHS we can apply inequality (2) if

$$
\frac{A_{0} A_{1}}{\alpha^{1 / p} b^{1 / p}} \cdot \frac{\beta+t}{p} \leq \frac{1}{2}
$$

But we defined $\beta$ for our convenience as a certain factor times $\alpha$. It is easy to see that taking for instance $t \leq \alpha$, we find the above expression to be $\sim \alpha^{1-\frac{1}{p}}$, and it is possible to make it sufficiently small if $p>1$ by choosing $\alpha$ small.

Now we can choose $\alpha$ such that the first term in the RHS is at least double the size of the second by (2) independently of $u$. Then we use (2) again and get

$$
\frac{A}{\alpha^{1 / p} b^{1 / p}}\left(\int\left|\nabla^{m} v\right|^{p} \tilde{d}_{\partial \Omega}(x)^{t} d x\right)^{\frac{1}{p}} \geq \sum_{k=0}^{m-1}\left(\int \frac{\left|\nabla^{k} u\right|^{p} \tilde{d}_{\partial \Omega}(x)^{\beta}}{\tilde{d}_{\partial \Omega}(x)^{(m-k) p}} d x\right)^{\bar{\nu}}
$$

but by (4)

$$
\mathrm{RHS} \geq \frac{1}{A} \cdot \sum_{k=0}^{m-1}\left(\int \frac{\left|\nabla^{k} v\right|^{p} \tilde{d}_{\partial \Omega}(x)^{t}}{\tilde{d}_{\partial \Omega}(x)^{(m-k) p}} d x\right)^{\frac{1}{p}}
$$

Hence the desired inequality is proved for the function $v$ since the tilde can be removed. But the class of functions we get from $C_{0}^{\infty}(\Omega)$ by multiplication with $\tilde{d}_{\partial \Omega}(x)^{-(\beta+t) / p}$ is of course the whole set $C_{0}^{\infty}(\Omega)$.

End of proof.

Corollary. Let $\Omega$ be an open and proper subset of $\mathbf{R}^{N}$ and let $p>1$. Consider the two conditions:

(i) $p>N$;

(ii) $\Omega$ is deformable to a point, and $p>N-1$.

Each condition implies that there is a positive $t_{0}$ which only depends on $m$, $p$, and $N$ such that for all $t<t_{0}$ and all $u \in C_{0}^{\infty}(\Omega)$ the following inequality is satisfied.

$$
\sum_{k=0}^{m-1} \int \frac{\left|\nabla^{k} u\right|^{p} d_{\partial \Omega}(x)^{t}}{d_{\partial \Omega}(x)^{(m-k) p}} d x \leq A \int\left|\nabla^{m} u\right|^{p} d_{\partial \Omega}(x)^{t} d x
$$

where $A$ is a constant depending only on $m, N, p$, and $t$.

Proof. The Maz'ja capacity:

$$
A \cdot \gamma_{m, m-1, p}\left(K, 2 Q_{0}\right) \geq C_{1, p}(K, 2 Q),
$$

where

$$
C_{1, p}(K, 2 Q)=\inf \left\{\|\nabla \varphi\|_{W^{1, p}}: \varphi \in C_{0}^{\infty}(2 Q),\left.\varphi\right|_{K} \geq 1\right\}
$$

by [7, p. 418]. But $C_{1, p}(K, 2 Q) \sim C_{1, p}(K)$,

$$
C_{1, p}(K)=\inf \left\{\|\varphi\|_{W^{1, p}}^{p}: \varphi \in C_{0}^{\infty},\left.\varphi\right|_{K} \geq 1\right\},
$$


which follows from the use of a cut-off function. For $p>1$ this capacity is equivalent to the Bessel capacity, for a definition see $[7, \S 7.2]$. This equivalence can be found in [11, p. 135].

This can also be derived from Lemma 2.1. in [4], which is a Poincaré inequality similar to that of Maz $^{\prime}$ ja (but independently derived). See also [13].

N. G. Meyers has proved in [9] that the Bessel capacity is nonincreasing after orthogonal projections. This has the effect that we can project the set $\widetilde{\Omega}^{c} \cap R_{\widetilde{Q}}$ surjectively on a line from the center of the cube to the boundary of the cube without getting a larger value of the capacity as a result.

Next is it known, see [7, chap. 7], that Bessel capacities $(p>1)$ are equivalent to Riesz capacities. Now [8] gives information on the metric properties of Riesz capacities, see Theorem 7.1. The information is given in the form that if a certain Hausdorff measure is positive (see [7] for a definition of Hausdorff measure), then a certain Riesz capacity is positive. The implications for the two cases in this corollary are that if $p>N$ then the Riesz capacity corresponding to $C_{1, p}$ is a positive constant for a point and if $p>N-1$ then the same capacity for line segment of fixed length is a positive constant.

This concludes the proof of the corollary.

\section{ACKNOWLEDGMENT}

I thank Lars Inge Hedberg who interested me in this subject and who had many discussions with me.

\section{REFERENCES}

1. A. Ancona, Une propriété des espaces de Sobolev, C. R. Acad. Sc. Paris Sér. I Math. 292 (1981), 477-480.

2. H. Brezis and F. E. Browder, Some properties of higher order Sobolev spaces, J. Math. Pures Appl. 61 (1982), 245-259.

3. G. H. Hardy, J. E. Littlewood, and G. Polya, Inequalities, Cambridge Univ. Press, Cambridge, 1952.

4. L. I. Hedberg, Two approximation problems in function spaces, Ark. Mat. 16 (1978), 51-81.

5. A. Kufner, Weighted Sobolev spaces, Teubner-Texte zur Math., Teubner, Leipzig, 1985.

6. V. G. Maz'ja, On $(p, l)$-capacity, imbedding theorems, and the spectrum of a selfadjoint elliptic operator, Math. USSR-Izv. 7 (1973), 357-387.

7. __ Sobolev spaces, Springer, Berlin-New York, 1985.

8. V. G. Maz'ja and V. P. Havin, Non-linear potential theory (in Russian). Uspekhi Mat. Nauk 27 (1972), 67-138. English translation: Russian Math. Surveys 27 (1972), 71-148.

9. N. G. Meyers, Continuity of Bessel potentials, Israel J. Math. 11 (1972), 271-283.

10. J. Nečas, Sur une méthode pour résoudre les équations aux dérivées partielle du type elliptique, voisine de la variationelle, Ann. Scuola Norm. Sup. Pisa Ser. 16, (1962), 305-326.

11. E. M. Stein, Singular integrals and differentiability of functions, Princeton Univ. Press, Princeton, NJ, 1970.

12. E. W. Stredulinsky, Weighted inequalities and degenerated elliptic partial differential equations, Springer-Verlag, New York.

13. A. Wannebo, Poincaré type inequalities for a cube and Hardy inequalities for a domain (in preparation).

Department of Mathematics, University of Stockholm, Stockholm, Sweden 